\title{
Uniform beam distributions at the target of the NASA Space Radiation Laboratory's beam line
}

\author{
N. Tsoupas, L. Ahrens, S. Bellavia, R. Bonati, K. A. Brown, I-Hung Chiang, C. J. Gardner, D. Gassner, S. Jao, \\ W. W. Mackay, I. Marneris, W. Meng, D. Phillips, P. Pile, R. Prigl, A. Rusek, L. Snydstrup, and K. Zeno \\ Brookhaven National Laboratory, P.O. Box 5000, Upton, New York 11973-5000, USA
}

(Received 22 November 2006; published 15 February 2007)

\begin{abstract}
Errors in delivering a uniformly distributed radiation dose to biological and material samples exposed to charged particle beams are a significant problem for experimenters. In this paper, we discuss data collected on the uniform beam distributions produced for NASA's Space Radiation Laboratory (NSRL) at Brookhaven National Laboratory (BNL), using a method that was conceived theoretically and tested experimentally at BNL. This method [N. Tsoupas et al., Nucl. Sci. Eng. 126, 71 (1997)] of generating uniform beam distributions on a plane normal to the beam's direction relies only on magnetically focusing the transported beam; no collimation of the beam is required or any other type of interaction of the beam with materials other than the target material. The method compares favorably with alternative methods of producing such distributions, and it can be applied to the entire energy spectrum of charged particle beams that are delivered to the NSRL's experiments by the Booster for the Alternating Gradient Synchrotron at BNL.
\end{abstract}

DOI: 10.1103/PhysRevSTAB.10.024701

PACS numbers: $29.20 .-\mathrm{c}, 41.85 .-\mathrm{p}$

\section{INTRODUCTION}

Researchers frequently require uniform irradiation of materials or biological samples by ion beams. At the NASA Space Radiation Laboratory (NSRL) at Brookhaven National Laboratory (BNL), we employ a unique method of generating uniformly irradiated areas ranging from $10 \times 10 \mathrm{~cm}^{2}$ to $20 \times 20 \mathrm{~cm}^{2}$ [1,2]. This facility, which started operations in 2003 [3], is used by NASA to uniformly irradiate various materials and biological samples with ion beams. Our method of generating uniform beam distributions at the target is based on magnetically focusing the transported beam. The only requirement that the beam must satisfy is that it must be distributed normally (i.e., Gaussian) in all transverse coordinates at the beginning of the transport line. In particular, the method does not require any collimation of the beam or interaction with any material other than the target material. To date, the facility has provided uniformly distributed beams of various ions including protons, ${ }^{12} \mathrm{C},{ }^{16} \mathrm{O},{ }^{28} \mathrm{Si},{ }^{39} \mathrm{Cl},{ }^{56} \mathrm{Fe}$, and ${ }^{48} \mathrm{Ti}$, at the target. These ion beams are first accelerated by the Booster of the Alternating Gradient Synchrotron (AGS) to various energies from 50 to $3000 \mathrm{MeV} / n$, then slowly extracted and transported via the NSRL's beamtransport line to the sample's location (target) which is $100 \mathrm{~m}$ downstream from the beginning of this line. The present method of producing uniformly distributed beams over a rectangular area normal to the beam's direction is based on third-order magnetic optics; it employs octupole magnetic elements specifically located along the beam line. These elements transform the otherwise normally distributed beam at the target into a uniformly distributed one. Details of this approach are given in Ref. [1], and the original experimental proof of the method appears in
Ref. [2]. In this paper, we describe the following: (a) the NSRL facility; (b) the NSRL beam-transport line; (c) the performance of this line in generating uniform beam distributions at the target's location (see Sec. IV); (d) theoretical calculations and measurements of the effects of misaligning the beam along the NSRL line upon the uniformity of its distribution at the target; e) theoretical calculations and experimental measurements of the effect of the beam coupling introduced by the octupole magnetic elements; (f) the possibility of using dodecapole magnetic elements, instead of octupole magnetic elements, to improve the uniformity of beam distribution at the target (see Sec. VII). Here, we define "beam distribution" at a particular point along the beam's direction as its distribution on a plane located at that particular point and normal to the beam's direction. The projection of the "beam's distribution" along the horizontal or vertical direction will be referred to, respectively, as the horizontal- or verticalbeam profile.

\section{THE NSRL FACILITY}

The construction of the NSRL facility at BNL was completed in March 2003, and the beam-transport line commissioned in June 2003 for use by NASA and other scientific organizations for experiments involving the irradiation of materials and biological samples [3,4]; part of NASA's research effort is to expose biological samples to a variety of ions similar to those that will be encountered on a spacecraft traveling to Mars. Figure 1 depicts the location of the NSRL facility in relation to the AGS Booster synchrotron, BNL's Tandem Van de Graff accelerator, and BNL's LINAC. The labels TtB and LtB in Fig. 1 designate the transport lines from the Tandem to the Booster, and the 


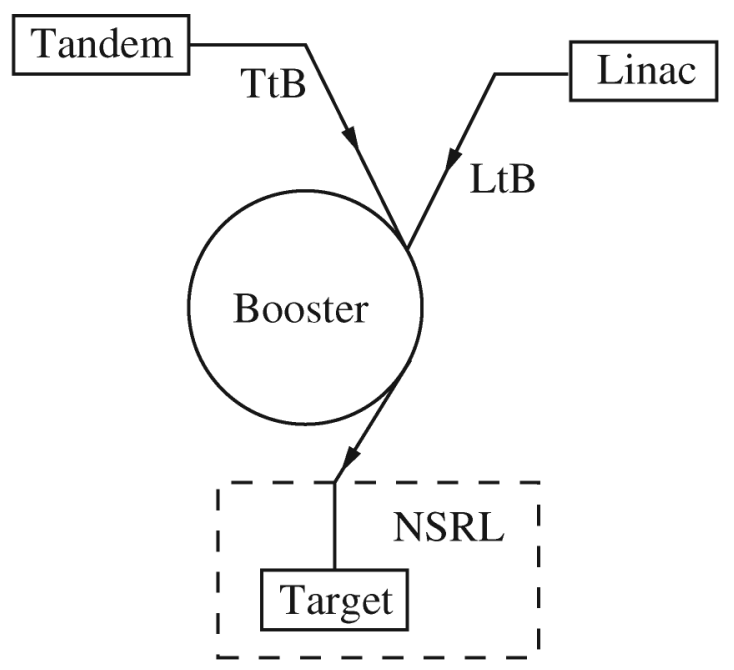

FIG. 1. Schematic diagram of the NSRL beam-transport line in relation to the AGS Booster synchrotron, the LINAC that provides a proton beam, and the Tandem accelerator that supplies beams of many different ions species. The Tandem to Booster (TtB) and the LINAC to Booster (LtB) transfer lines are shown in the figure.

LINAC to the Booster, respectively. The line originating from the Booster ring and terminating on the TARGET is the NSRL beam-transport line.

The ion beams used at the NSRL facility to irradiate samples are produced by BNL's Tandem (Van de Graaff), which provides many light- and heavy-ion species, or BNL's LINAC, which supplies protons only. The ion beams are transported to, and accelerated by, the AGS Booster synchrotron, and subsequently are slowly extracted [4] into the NSRL beam-transport line to deliver a uniform beam distribution at the sample. Column 1 of Table I shows the ion beams that have been accelerated and transported since operations began at the facility. Column 2 shows the various energies to which each ion was accelerated before its transport. Column 3 shows the charged state of each ion during acceleration in the AGS Booster synchrotron. The labels $Q_{b s}$ and $Q_{a s}$ are the charge

TABLE I. Ion species and energies of the ions, which have been transported via the NSRL beam-transport line since the start of NSRL operations. The labels $Q_{b s}$ and $Q_{a s}$ are the charge states of the ions before and after the stripping foil (see text).

\begin{tabular}{ccccc}
\hline \hline \multicolumn{3}{c}{ Energy } \\
Ion & {$[\mathrm{MeV} / n]$} & $Q_{b s}$ & $Q_{a s}$ & $\begin{array}{c}\text { Ions/cycle } \\
10^{9}\end{array}$ \\
\hline$p$ & $2500,730,500,200$ & +1 & +1 & 34 \\
${ }^{12} \mathrm{C}$ & 300 & +5 & +6 & 12 \\
${ }^{16} \mathrm{O}$ & 1000 & +7 & +8 & 5 \\
${ }^{28} \mathrm{Si}$ & 1000,300 & $+9,+13$ & +14 & 3 \\
${ }^{39} \mathrm{Cl}$ & 550 & +14 & +17 & 2 \\
${ }^{48} \mathrm{Ti}$ & 1000,300 & +18 & +22 & 0.8 \\
${ }^{56} \mathrm{Fe}$ & 1000,600300 & +20 & +26 & 2 \\
\hline \hline
\end{tabular}

states of the ions, before and after the stripping foil, discussed below.

An ion beam usually is stripped of its electrons before being transported to the target by a $0.051 \mathrm{~mm}$ thick copper foil sited at the entrance of the NSRL line; it can be inserted and retracted automatically from the beam's path. Column 4 of Table I gives the charge state of the ion after stripping. Electron stripping offers two benefits: first, it reduces the rigidity of the transported beam, and second, the multiple scattering of the ion beam at the foil enforces one of the requirements for generating a uniform beam distribution. This requirement is that the beam, which is slowly extracted from the AGS Booster synchrotron, is normally distributed at the entrance of the NSRL beam-transport line. The last column of Table I shows the typical number of ions delivered at the target per Booster cycle.

\section{THE NSRL BEAM-TRANSPORT LINE}

\section{A. Magnetic elements}

The magnetic elements of the NSRL's beam-transport line are the "main magnets," viz. the bending dipoles, the quadrupoles, and the octupoles that bend and focus the

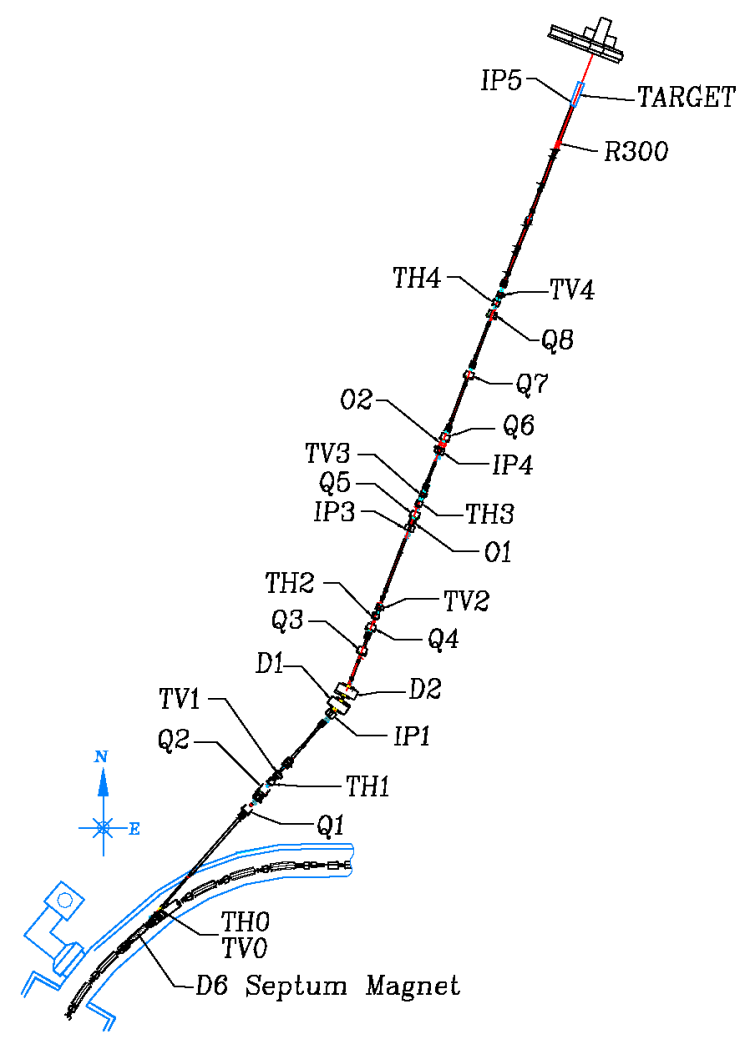

FIG. 2. (Color) The NSRL beam-transport line showing the main magnets (dipoles quadrupoles octupoles), the corrector magnets (TH, TV), and the instrumentation packages (IP). The sector appearing at the bottom of the figure is part of the Booster synchrotron ring. 
transported beam, and the "corrector magnets," which are the horizontal- and vertical-corrector dipoles that correct the beam's trajectory in the beam line. Figure 2 shows the layout of the magnetic elements of the transport line. The arc at the bottom of the figure is part of the AGS Booster synchrotron ring.

Table II lists the magnetic elements of the NSRL line and some of their properties. Starting with the first column, the multipole type of each magnetic element is given, followed by its physical length, then the magnet gap of the dipoles or the radius of the aperture of the quadrupoles or the octupoles, the maximum allowed current of the magnet's coil, the number of coils per magnet, the number of turns per coil, and finally, in the last column on the left, the integrated strength $S=\int\left\{B_{\text {pole }} / R^{n}\right\} d l$ of each element. The symbols $B_{\text {pole }}$ and $R$ inside the integral, above, are the $B$ field at the pole face, and the aperture radius of the magnetic element, respectively. The integral is taken along the beam's direction. The strength $S$ for a dipole corresponds to $n=0$, for a quadrupole to $n=1$, and for an octupole to $n=3$. The coils of the dipoles D1 and D2, shown in Table II and in Fig. 2, are connected in series to a single power supply, to form a dipole with a total bending angle of $20^{\circ}$. The horizontal-corrector and verticalcorrector dipoles that correct the beam's horizontal and vertical trajectory also are listed in Table II. The D6 magnet is a current septum magnet with a maximum allowed current density in the septum coil of $\sim 8 \mathrm{kA} / \mathrm{cm}^{2}$. When the D6 septum is excited at the maximum current density it can bend ion beams with rigidity of $13 \mathrm{~T} \mathrm{~m}$ by an angle of $9.8^{\circ}$.

\section{B. Beam diagnostics}

Five instrumentation packages (IP) [5] installed at specified locations along the beam line allow us to characterize the transported beam. Figure 2 shows their relative locations. Each package is equipped with an ionization chamber (IC) to measure the beam's intensity, and a segmented wire ionization chamber (SWIC) that measures the horizontal- and vertical-beam profiles. The devices IC and SWIC are inserted into the path of the beam to measure its corresponding properties. Additional diagnostic devices we inserted are the phosphor visual flags (VF) that measure the beam's distribution as well as its horizontal and vertical profiles. VF are located $0.75 \mathrm{~m}$ upstream of each instrumentation package, one near the end of the beam-transport line (R300, in Fig. 2) and also at the target.

\section{Beam constraints of the transport line}

Apart from the constraints that a beam line must satisfy to be compatible with the topology of the building and the size of the beam pipe, additional ones were imposed on the optics of the NSRL beam-transport line: (a) The septum

TABLE II. The magnetic elements of the NSRL beam-transport line, and some of their properties. The relative location of the magnetic elements is shown in Fig. 2.

\begin{tabular}{|c|c|c|c|c|c|c|}
\hline Element name/multipole & $\begin{array}{c}L \\
{[\mathrm{~m}]}\end{array}$ & $\begin{array}{c}\mathrm{Gap} / R \\
{[\mathrm{~cm}]}\end{array}$ & $\begin{array}{c}I \\
{[\mathrm{kA}]}\end{array}$ & Coils & Turns/coil & $\begin{array}{c}S \\
{[\mathrm{~T} \mathrm{~m}],[\mathrm{T}],\left[\mathrm{T} \mathrm{m}^{2}\right]}\end{array}$ \\
\hline D6 $9.8^{\circ}$ dipole septum magnet & 2.525 & 2.75 & 5.0 & 1 & 4 & 2.14 \\
\hline TV0 vertical dipole corrector & 0.203 & 13.0 & 0.05 & 2 & 52 & 0.008 \\
\hline TH0 horizontal dipole corrector & 0.203 & 13.0 & 0.05 & 2 & 52 & 0.008 \\
\hline Q1 quadrupole magnet & 0.711 & 10.16 & 2.7 & 4 & 22 & 9 \\
\hline Q2 quadrupole magnet & 0.711 & 10.16 & 2.7 & 4 & 22 & 9 \\
\hline TH1 horizontal dipole corrector & 0.40 & 22.98 & 0.5 & 2 & 32 & 0.098 \\
\hline TV1 vertical dipole corrector & 0.400 & 22.98 & 0.5 & 2 & 32 & 0.098 \\
\hline D1 $10^{\circ}$ left-bend dipole & 1.15 & 15.25 & 3.5 & 2 & 60 & 2.542 \\
\hline D2 $10^{\circ}$ left-bend dipole & 1.15 & 15.25 & 3.5 & 2 & 60 & 2.542 \\
\hline Q3 quadrupole magnet & 0.711 & 10.16 & 2.7 & 4 & 22 & 9 \\
\hline Q4 quadrupole magnet & 0.711 & 10.16 & 2.7 & 4 & 22 & 9 \\
\hline $\mathrm{TH} 2$ horizontal dipole corrector & 0.400 & 22.98 & 0.5 & 2 & 32 & 0.098 \\
\hline TV2 vertical dipole corrector & 0.400 & 22.98 & 0.5 & 2 & 32 & 0.098 \\
\hline O1 horizontal defocusing octupole & 0.500 & 10.16 & 2.0 & 8 & 8 & 382.8 \\
\hline Q5 quadrupole magnet & 0.711 & 10.16 & 2.7 & 4 & 22 & 9 \\
\hline TH3 horizontal dipole corrector & 0.400 & 22.98 & 0.5 & 2 & 32 & 0.098 \\
\hline TV3 vertical dipole corrector & 0.400 & 22.98 & 0.5 & 2 & 32 & 0.098 \\
\hline O2 horizontal focusing octupole & 0.500 & 10.16 & 2.0 & 8 & 8 & 382.8 \\
\hline Q6 quadrupole & 0.711 & 10.16 & 2.7 & 4 & 22 & 9 \\
\hline Q7 quadrupole & 0.711 & 10.16 & 2.7 & 4 & 22 & 9 \\
\hline Q8 quadrupole & 0.711 & 10.16 & 2.7 & 4 & 22 & 9 \\
\hline TH4 horizontal dipole corrector & 0.400 & 22.98 & 0.5 & 2 & 32 & 0.098 \\
\hline TV4 vertical dipole corrector & 0.400 & 22.98 & 0.5 & 2 & 32 & 0.098 \\
\hline
\end{tabular}


TABLE III. The theoretical and measured beam parameters at the beginning of the NSRL beam-transport line. ("n/meas" is "not measured."')

\begin{tabular}{|c|c|c|c|c|c|c|c|c|}
\hline & $\alpha_{x}$ & $\beta_{x}[\mathrm{~m}]$ & $\eta_{x}[\mathrm{~m}]$ & $\eta_{x}^{\prime}$ & $\alpha_{y}$ & $\beta_{y}[\mathrm{~m}]$ & $\eta_{y}[\mathrm{~m}]$ & $\eta_{y}^{\prime}$ \\
\hline Theory & 1.87 & 10.0 & 0.0 & 0.0 & 0.637 & 4.39 & 0.0 & 0.0 \\
\hline Experiment & $0.3 \pm 0.2$ & $10.6 \pm 3.0$ & $\mathrm{n} / \mathrm{meas}$ & $\mathrm{n} /$ meas & $-0.51 \pm 0.2$ & $7.4 \pm 1.2$ & $\mathrm{n} / \mathrm{meas}$ & $\mathrm{n} / \mathrm{meas}$ \\
\hline
\end{tabular}

magnet D6, the quadrupoles Q1, Q2, and the dipoles D1, D2 (shown in Fig. 2) form an achromatic bend. Therefore, at the exit of the $\mathrm{D} 2$ dipole magnet the beam is achromatic, provided that it also is achromatic at the entrance of the D6 septum magnet. (b) The $\left(x, x^{\prime}\right)$ particle coordinates of the beam at the location of the octupole (O1) are highly correlated [1]. This constraint is required to facilitate the effect of the octupole magnetic element, which when excited generates a uniform beam distribution at the location of the target along the horizontal direction. (c) A similar constraint, of highly correlated beam coordinates $\left(y, y^{\prime}\right)$ also must be satisfied at the location of the second octupole $(\mathrm{O} 2)$ to ensure the beam's uniformity at the target along the vertical direction.

\section{First-order beam optics}

The first-order beam optics satisfying these beam constraints (a), (b), and (c) was implemented by modeling the transport line using the computer code TRANSPORT [6]. In the model, we assumed that the beam parameters $\left(\alpha_{x, y}\right.$, $\left.\beta_{x, y}\right)$ and dispersion functions $\left(\eta_{x, y}\right)$ of the extracted beam at the beginning of the NSRL line are identical to those of the circulating beam in the AGS Booster synchrotron just before extraction. Table III gives the beam's calculated (2nd row) and experimental (3rd row) horizontal and vertical parameters at the start of the NSRL line.

These parameters were used in designing the NSRL line to generate a uniform distribution of the beam on the target. We assumed a normal distribution of the beam in all of its coordinates at the beginning of the NSRL line. In fact, to enforce the requirement that the slowly extracted beam acquires a normal distribution there, it is passed through a thin $\mathrm{Cu}$ foil which is inserted at the entrance of the D6 septum magnet. A set of thin $\mathrm{Cu}$ foils or single thin tungsten $(\mathrm{W})$ wires are mounted on a wheel which is placed at the entrance of the D6 septum magnet. During normal operations, the wheel is rotated to place a particular $\mathrm{Cu}$ foil or a single W wire in the beam's path. The effect of the foil or wire on the beam is twofold; first, to reduce the rigidity of the transported beam by stripping the ions of the electrons, and second, to ensure that the beam acquires a Gaussian distribution through the multiple scattering of the ions in the foil. We usually insert into the beam the $\mathrm{Cu}$ foils of $0.51 \mathrm{~mm}$ or $0.051 \mathrm{~mm}$ thickness, depending on the ion species and beam's momentum. Table I shows the charge states, before and after stripping, of some of the ions transported by the NSRL line. Figure 3 plots the horizontal and vertical beta $\left(\beta_{x}, \beta_{y}\right)$ and dispersion $\left(\eta_{x}, \eta_{y}\right)$ functions along the NSRL beam-transport line for a particular beam focusing that satisfies the constraints for having a highly correlated beam at the octupoles. The computer code TRANSPORT [6] was used to calculate the functions $\left(\beta_{x}, \beta_{y}\right)$ and $\left(\eta_{x}, \eta_{y}\right)$ plotted in Fig. 3 which also shows the relative locations of the magnetic elements (blue boxes) along the transport line.

This particular approach to focus the beam will generate at the target located $100 \mathrm{~m}$ downstream from the start of the NSRL line, a uniformly distributed beam with a rectangular cross section of $20 \times 20 \mathrm{~cm}^{2}$, provided that both octupole magnetic elements are excited. Table IV gives the strength $\int\{B d l\} /(B \rho)$ of the dipole in $\mathrm{mrad}$ and the strength $\int\{G d l\} /(B \rho)$ of the quadrupole-magnetic elements in $\mathrm{m}^{-1}$ that were used in modeling the NSRL beam-transport line to calculate the beam parameters $\left(\beta_{x}, \beta_{y}\right)$ and $\left(\eta_{x}, \eta_{y}\right)$ shown in Fig. 3.

The symbols $B \rho, \int B d l$, and $\int G d l$ in the preceding text represent the beam's rigidity, the integrated dipole field, and integrated field gradient along the dipole- and quadrupole-magnetic elements, respectively. The beam profiles in Fig. 4 are generated from the beta $\left(\beta_{x}, \beta_{y}\right)$ and dispersion $\left(\eta_{x}, \eta_{y}\right)$ functions shown in Fig. 3 using the

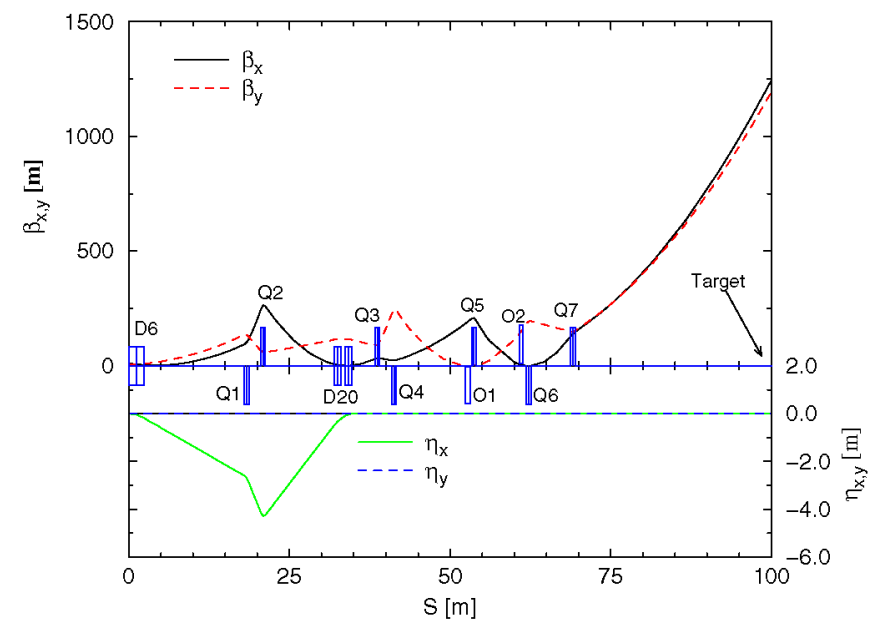

FIG. 3. (Color) The horizontal and vertical beta $\left(\beta_{x}, \beta_{y}\right)$ and dispersion $\left(\eta_{x}, \eta_{y}\right)$ functions along the NSRL beam-transport line. The rectangular blue boxes in the figure correspond to the magnetic elements of the line and the label next to each element is its name. Note that the vertical dispersion function $\eta_{y}$, along the line is zero. The label D20 in the figure designates the two dipoles D1 and D2 (Table II). 
TABLE IV. The strength of the magnetic elements corresponding to the first-order beam optics of the NSRL line used to generate uniform beam at the target when the octupoles are ON.

\begin{tabular}{ccccccccc}
\hline \hline $\begin{array}{c}\text { D6 } \\
{[\mathrm{mrad}]}\end{array}$ & $\begin{array}{c}\mathrm{Q} 1 \\
{\left[\mathrm{~m}^{-1}\right]}\end{array}$ & $\begin{array}{c}\text { Q2 } \\
{\left[\mathrm{m}^{-1}\right]}\end{array}$ & $\begin{array}{c}\mathrm{D} 1 / \mathrm{D} 2 \\
{[\mathrm{mrad}]}\end{array}$ & $\begin{array}{c}\text { Q3 } \\
{\left[\mathrm{m}^{-1}\right]}\end{array}$ & $\begin{array}{c}\text { Q4 } \\
{\left[\mathrm{m}^{-1}\right]}\end{array}$ & $\begin{array}{c}\text { Q5 } \\
{\left[\mathrm{m}^{-1}\right]}\end{array}$ & $\begin{array}{c}\text { Q6 } \\
{\left[\mathrm{m}^{-1}\right]}\end{array}$ & $\begin{array}{c}\text { Q7 } \\
{\left[\mathrm{m}^{-1}\right]}\end{array}$ \\
\hline 155.3 & 0.1891 & 0.2393 & 174.53 & 0.3007 & 0.2537 & 0.1755 & 0.1309 & 0.0819 \\
\hline \hline
\end{tabular}

relation $\sigma_{x, y}=\left\{\beta_{x, y} \cdot \varepsilon_{x, y}+\left(\eta_{x, y} \cdot \delta p / p\right)^{2}\right\}^{1 / 2}$. We used a 95\% normalized beam emittance $\left(\varepsilon_{x, y}\right)$ of $10 \pi \mathrm{mm}$ mrad in determining the beam profiles $\left(\sigma_{x, y}\right)$ shown in Fig. 4; accordingly, they contain $98.5 \%$ of the beam's intensity. A $98.5 \%$ beam momentum spread $\delta p / p$ of $0.5 \%$ also was used in the calculations.

We also assessed experimentally the beam's parameters at the beginning of the NSRL line and the beam emittance by measuring the horizontal- and vertical-beam profiles along the line at the SWIC locations. Subsequently, from these measurements, the beam's emittances $\varepsilon_{x, y}$ and parameters $\left(\beta_{x, y}, \alpha_{x, y}\right)$ can be calculated at the beginning of the NSRL line. For example, Table III, third row, lists the experimentally measured beam parameters of a $\mathrm{Ti}$ beam with kinetic energy of $1.0 \mathrm{GeV} /$ nucleon after passing through a $0.51 \mathrm{~mm} \mathrm{Cu}$ foil. Its measured projected beam emittances (95\% normalized) were, respectively, $\varepsilon_{x}=$ $4.3 \pm 1.0 \pi \mathrm{mm} \mathrm{mrad}$ and $\varepsilon_{y}=2.6 \pm 0.5 \pi \mathrm{mm} \mathrm{mrad}$ for the horizontal and vertical plane. The theoretically calculated beam parameters in Table III, second row, are not within the errors of the experimentally measured ones shown in the third row. We offer two reasons for this discrepancy between them, but there may be several. First, we have assumed in designing the NSRL line to generate uniform beam distributions that the theoretical beam parameters of the slowly extracted beam at the

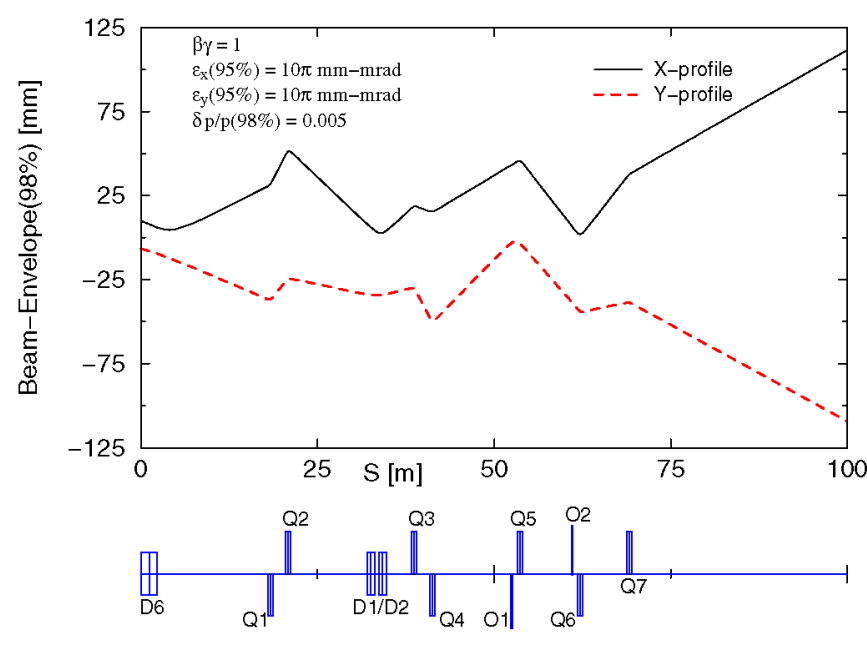

FIG. 4. (Color) Half of the horizontal (black solid line) and half of the vertical (red dashed line) beam profile of the beam transported down the NSRL line. The beam profiles contain $98.5 \%$ of the beam's intensity. As in Fig. 3, the main magnetic elements of the line with their names appear at the bottom of the plot. entrance of the NSRL line are the same as those of the circulating beam; this assumption may be not totally correct since the beam parameters of the extracted beam might be affected by the slow extraction process devices, which were not included in the model which described the circulating beam in the AGS Booster synchrotron. Also, the strength of the extraction devices varies during the extraction process, causing changes in the beam's parameters at the beginning of the NSRL line. A second reason for the discrepancy might have resulted from our basing the theoretical calculations on the supposition that all coordinates of the beam are distributed normally. Rather, this may be an approximation since the slow extraction process employs sextupoles that can alter a normally distributed beam.

\section{THIRD-ORDER BEAM OPTICS}

The first-order beam optics, which satisfies the constraints discussed earlier, provides the conditions for the octupoles to transform the "normally" distributed beam at the target into a beam, with rectangular cross section, that is uniformly distributed over this rectangle. The following equation was used to calculate the beam's distribution at the target:

$$
X_{i}=R_{i j} x_{j}+T_{i j k} x_{j} x_{k}+W_{i j k l} x_{j} x_{k} x_{l}
$$

where $x_{i}$, and $X_{i}$ correspond to the particle coordinates at the entrance and exit, respectively, of the beam's transport line, and the index $(i)$ designates a particular particle coordinate according to relation (2) below:

$$
\left(x_{1}, x_{2}, x_{3}, x_{4}, x_{5}, x_{6}\right) \Leftrightarrow\left(x, x^{\prime}, y, y^{\prime}, d l / l, \delta p / p\right) .
$$

The quantities $R_{i j}, T_{i j k}$, and $W_{i j k l}$ in Eq. (1) are the first-, second-, and third-order aberration coefficients, respectively, of the beam line. Each index runs from 1 to 6 according to relation (2), assuming the summation is performed over the repeated indices of each term in Eq. (1). The aberration coefficients depend on the location, multipolarity, and strength of each element. The aberration coefficients of the beam line and the beam's distribution $\left\{x_{i}\right\}$ at the entrance of the transport line uniquely define its distribution $\left\{X_{i}\right\}$ at the exit of the beam line. In Eq. (1), which is the Taylor series expansion of the $X_{i}$ particle coordinates at the exit as a function of the particle coordinates $x_{i}$ at the entrance, we omitted the aberration coefficients of order higher than three. Reference [1] details 

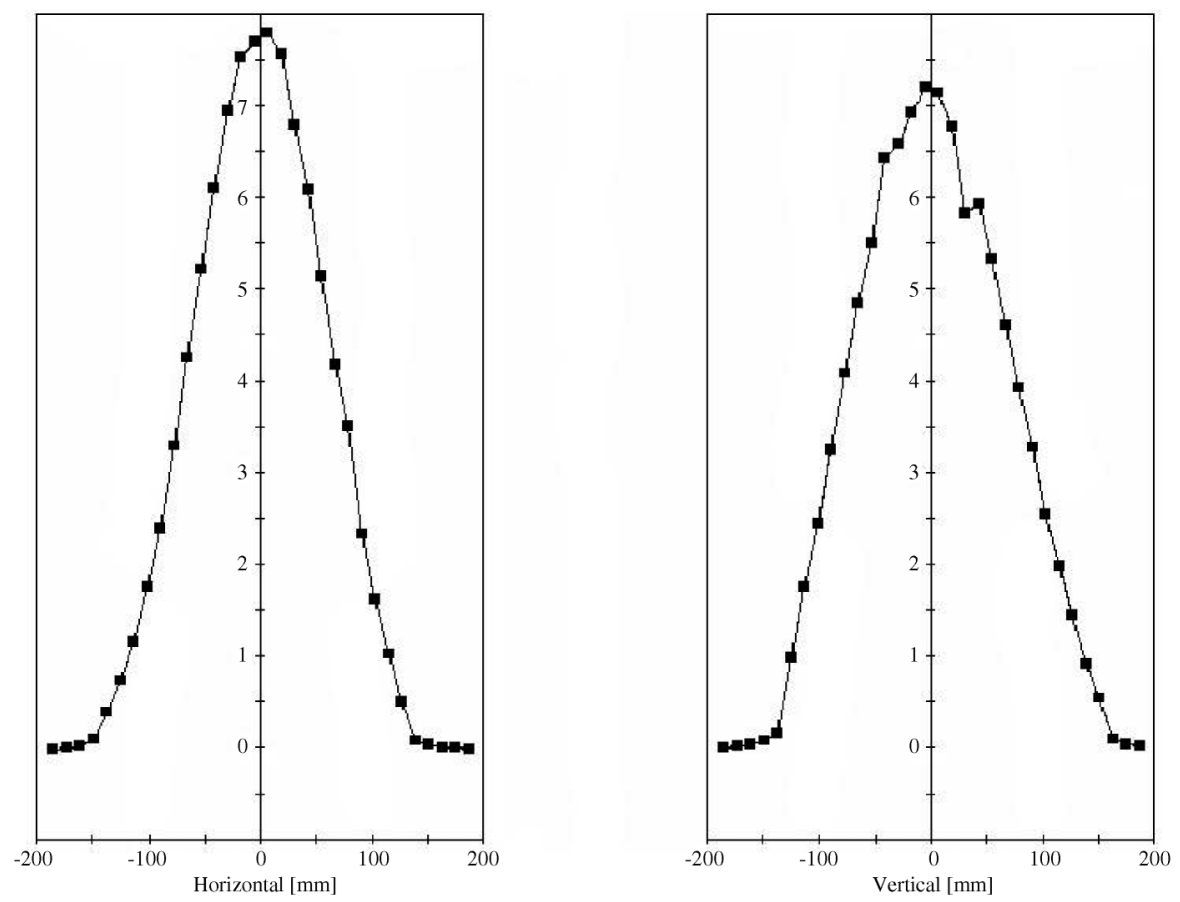

FIG. 5. The horizontal and vertical projections of the beam's distribution at the target location of the NSRL beam-transport line when the octupoles shown in Figs. 2 and 3 are OFF. These beam profiles correspond to the line's first-order beam optics which satisfies the beam constraints discussed in the Sec. IIID.

the theory describing the action of the octupoles on the beam distribution, which is assumed to be "normal" in all of its coordinates, at the entrance of the beam line. Here, we present only the experimental measurements of the beam's distributions at the target, with the octupoles turned $\mathrm{ON}$ and OFF. Figure 5 shows the horizontal and vertical projections of these distributions at the target when the octupoles are OFF.
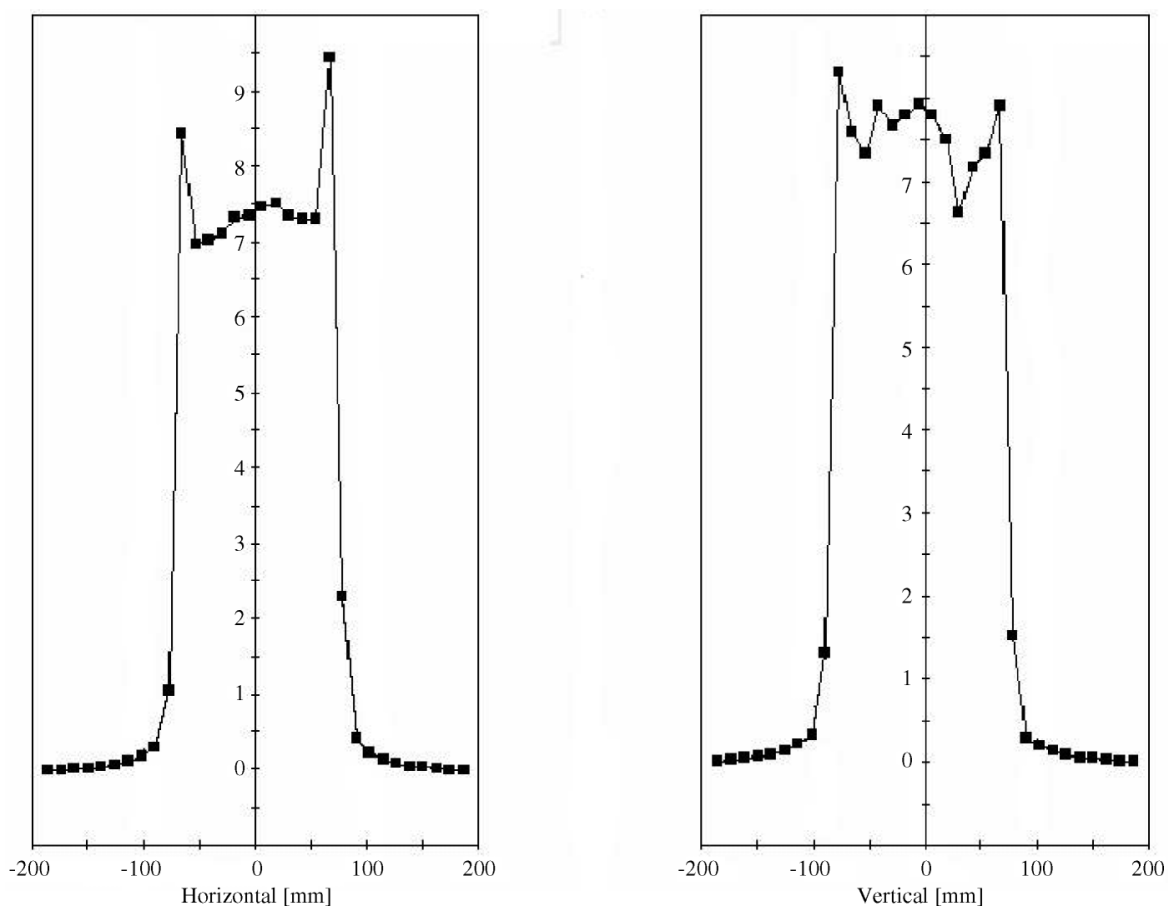

FIG. 6. The horizontal and vertical projections of the beam's distribution at the NSRL target location when the octupoles O1, O2, shown in Figs. 3 and 4 are ON. 
These nearly Gaussian beam distributions were obtained with the SWIC that is located at the target. Figure 6 illustrates the corresponding, horizontal and vertical projections of the distribution (as shown in Fig. 5), at the target but with the octupoles of the beam line turned ON. A comparison of the beam profiles shown in Fig. 5 with those shown in Fig. 6 indicates the strong effect of the octupoles on the beam distribution at the target. The sudden dip in this distribution, shown by the SWIC's vertical wire located at a distance $\sim 45 \mathrm{~mm}$, does not reflect a real decrease in the beam's intensity, but rather an incorrect setting of electronic gain of this wire.

However, the increase of the beam intensity shown at the edges of the distribution in Fig. 6 is real, characteristic of the effect of the octupole magnetic elements on the beam's distribution, as explained later. The SWICs along the transport line measure the horizontal- and vertical-beam profiles at lower beam intensities, and at lower spatial resolution than do the visual flags. The latter, however, can measure both the distribution and its horizontal and vertical projections at each of their locations; they appear in Figs. 7(a)-7(d). The beam image shown in Fig. 7(a) has been obtained with the R300 visual flag shown in Fig. 2. The variation of the beam intensity in the inner part $\left(\sim 19 \times 17 \mathrm{~cm}^{2}\right)$ of this rectangular image is $\pm 2.5 \%$. At the edges of the rectangle the beam intensity is higher. The image distortion (base side of the rectangle is longer than the top side) is not due to any midplane asymmetry of either the magnetic elements of the NSRL line or any asymmetry of the beam distribution, but is due to the optics which transports the light from this visual flag to the CCD of the camera.

Figure 7(b) shows another rectangular beam image of dimensions $20 \times 20 \mathrm{~cm}^{2}$. It was obtained with a different visual flag that moves on a system of rails, and can be positioned various distances downstream from the last quadrupole element, Q8, of the NSRL line. The optical system being used to capture the image from this flag is of a "periscope" type, free from the distortion in the image of the R300 flag. The beam's uniformity within the $19 \times 19 \mathrm{~cm}^{2}$ area of the image is well within $\pm 2.5 \%$. The images in Figs. 7(a) and 7(b) reveal that the beam's

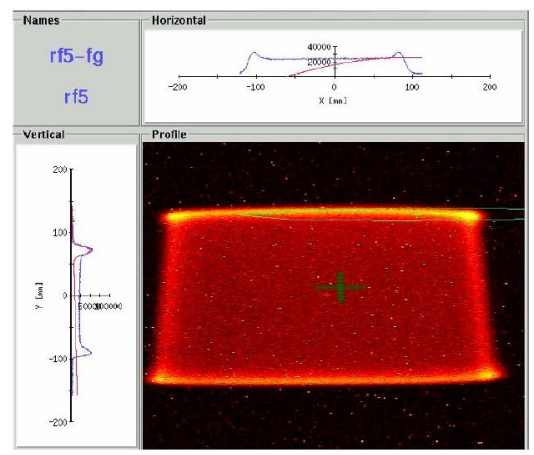

(a)

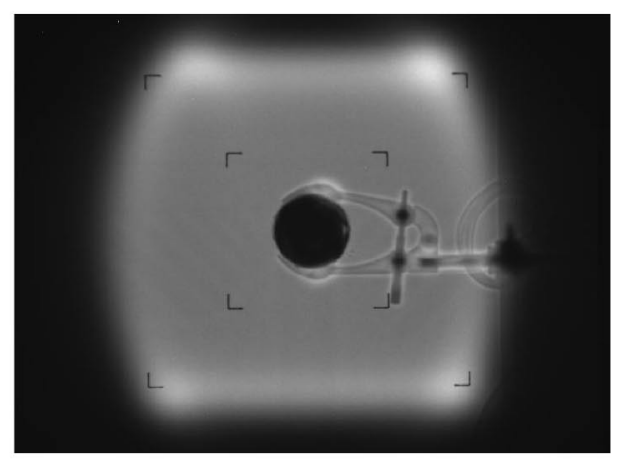

(c)

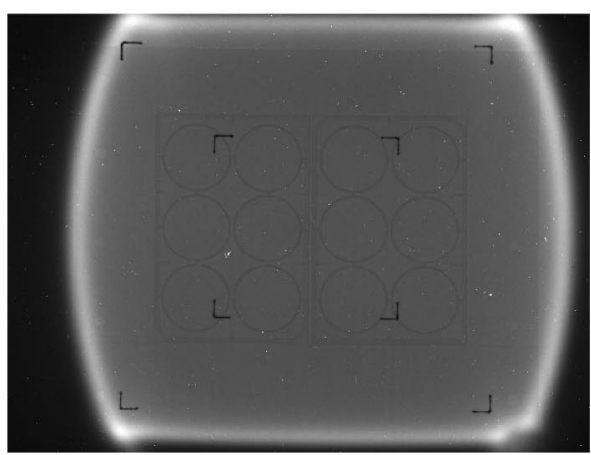

(b)

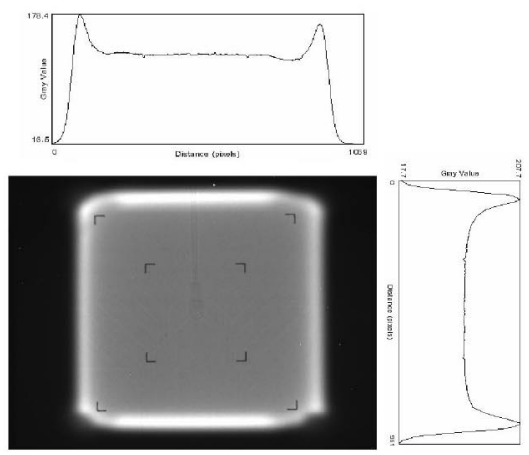

(d)

FIG. 7. (Color) (a) An image of the beam's distribution at the target location of the NSRL beam-transport line, obtained with a visual flag. The octupoles are ON. We note that the bottom side of the nearly rectangular image is larger than the top; this distortion is due to optic transport of the light from the visual flag to the camera. The blue curves on the top and the left side of the figure are the plots of the horizontal and vertical beam profiles of the beam distribution. (b) A beam distribution at the target location of the NSRL line, obtained with a visual flag. The octupoles of the line are turned ON, to generate a uniformly irradiated area within a large portion of the rectangular area of this distribution. (c) Another image of a beam distribution at the target location of the NSRL line, obtained with a visual flag. The sample under irradiation is shown as a dark circle. The sample holder also is visible. (d) A beam's distribution at the target location of the NSRL line. The horizontal and vertical projections of the distribution (beam profiles) appear on the top and the right side of the figure. 
intensity falls quickly to zero outside the rectangular radiation areas. We explain the higher intensity at the edges of the distributions in a later section. We also note that the images in these figures are not perfect rectangles since their sides are curved. However, this defect does not alter the size of the area where the beam is uniform.

The curved sides of the images are due to the focusing of the beam, and not to its "collimation" by the vacuum pipe, as we demonstrated in detailed experimental studies that included measuring the beam's intensity along the transport line and also its lateral motion using the corrector dipole magnets. There were no beam losses along the beam line that could cause these curved sides. To understand their origin, we recall that one constraint imposed in the first-order beam optics is that the $\left(x, x^{\prime}\right) /\left(y, y^{\prime}\right)$ coordinates of the beam at the horizontal/vertical octupole should be highly correlated. This constraint minimizes the effect of aberration coefficients such as $W_{1133}, W_{1134}$, and $W_{1144}$ that couple the horizontal- and vertical-beam coordinates, so allowing the $W_{1111}$ and $W_{3333}$ coefficients to dominate. Computer simulations demonstrated that, by either relaxing the constraint of the highly correlated beam, or by increasing the size of the horizontal- or vertical-beam size at the octupoles, we can generate curved sides on the beam's distributions similar to those in Figs. 7(a) and 7(b). Thus, one reason why the sides of the beam distributions are curved is the effect of aberration coefficients, which couple the horizontal- and vertical-beam coordinates, under conditions wherein the beam is not sufficiently highly correlated or large enough to approach the aperture of the octupole. In another computer simulation, we modified the normal distribution of the beam at the entrance of the NSRL line by increasing its horizontal intensity only at the edges of the original normal distribution. This change resulted in curved sides of the beam distribution at the target. Figure 7(c) is another beam image, obtained with the same visual flag as that shown in Fig. 7(b).

Similarly, Fig. 7(d) is another beam distribution with its horizontal and vertical projections (beam profiles) in the top and the right sides. We note that the increased intensity at the perimeter of the rectangular radiation area also causes a rise in the beam's intensity at the edges of the projected horizontal and vertical distributions.

\section{EFFECTS OF BEAM MISALIGNMENT ON THE UNIFORMITY OF THE BEAM'S DISTRIBUTION AT THE TARGET}

Our standard setup of the NSRL line that ensures a uniform distribution of the beam at the target is not always successful. Then, the profiles at the target may look similar to those depicted in Fig. 8.

These projected distributions, especially the horizontal one, clearly show that the beam's distribution at the target is not uniform. Such nonuniform profiles indicate that the beam's center line does not coincide with the magnetic axis of the octupoles that is responsible for modifying the horizontal or vertical distribution of the beam at the target.

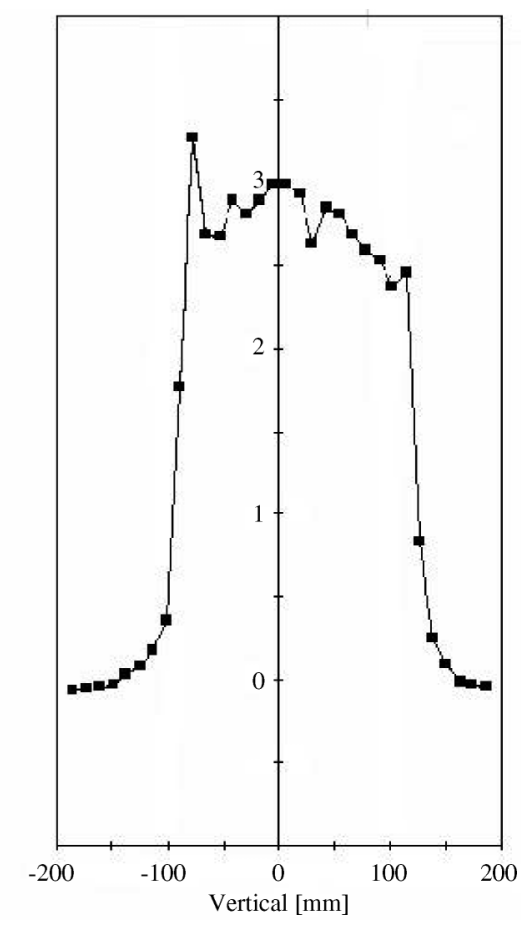

FIG. 8. Measured, horizontal and vertical projections of the beam's distribution at the target location with the octupoles ON. These beam profiles were generated by displacing horizontally, at octupole O1, the beam's axis by $5 \mathrm{~mm}$ with respect to the axis of the octupole.

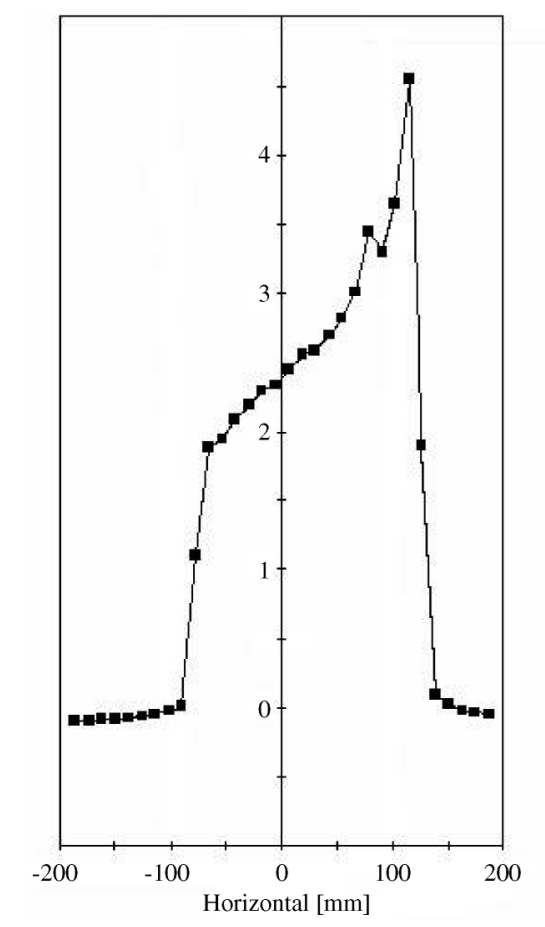




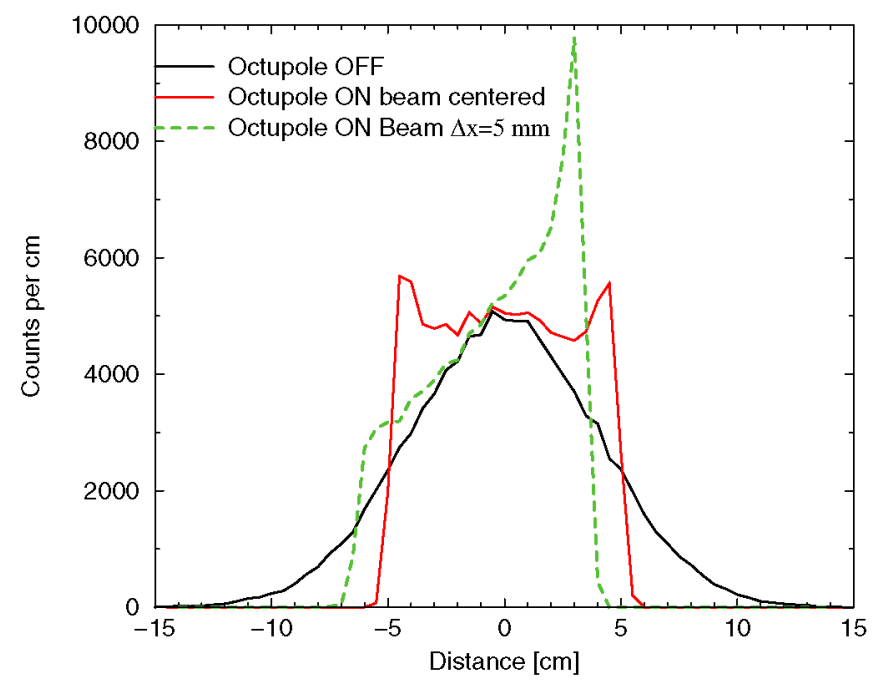

FIG. 9. (Color) Computer simulations of horizontal beam profiles on the target for three different cases (see text). The parallel displacement of the octupole's axis from the center of the beam generates the "slanted" beam distribution (green dotted line) on the target.

This misalignment can be corrected by adjusting a horizontal- and/or vertical-corrector magnet upstream of the octupole to place the beam along the octupole's magnetic axis. Computer simulations can reproduce nonuniformities in the beam profile like that in Fig. 8 by simply displacing the corresponding magnetic axis of the octupole from the center line of the beam or vice versa. The results from such simulations in Fig. 9 show the three curves corresponding to (a) the horizontal projection of the beam's distribution at the target location when the octupole that affects it is OFF, therefore the beam's profile is normally distributed, (b) the horizontal projection of the beam's distribution at the target when the octupole that affects it is ON, therefore, this profile is rectangular, and the beam's central ray coincides with the octupole's center, (c) the horizontal projection of the beam's distribution at the target when the octupole that affects it is ON but misaligned with respect to the beam axis. This profile is asymmetric and exhibits a slope because the "horizontal" octupole is ON and the octupole's magnetic axis is displaced horizontally by $5 \mathrm{~mm}$ with respect to the beam's central ray.

Each of the beam profiles shown in Fig. 9 is the projection along the horizontal axis of each of the phase-space $\left(x, x^{\prime}\right)$ plots shown in Fig. 10.

In particular, when the horizontal octupole is OFF, the distribution in phase space $\left(x, x^{\prime}\right)$ (black dots in Fig. 10) forms a "thin" ellipse, as expected. The projection of this distribution on the $x$-axis is the Gaussian- shaped curve in Fig. 9. When the octupole is ON, it transforms the elliptical phase-space distribution into one where the edges of the ellipse are folded towards the center (red dots); its projection on the $x$-axis generates the symmetric rectangular beam distribution shown in Fig. 9. When the octupole is

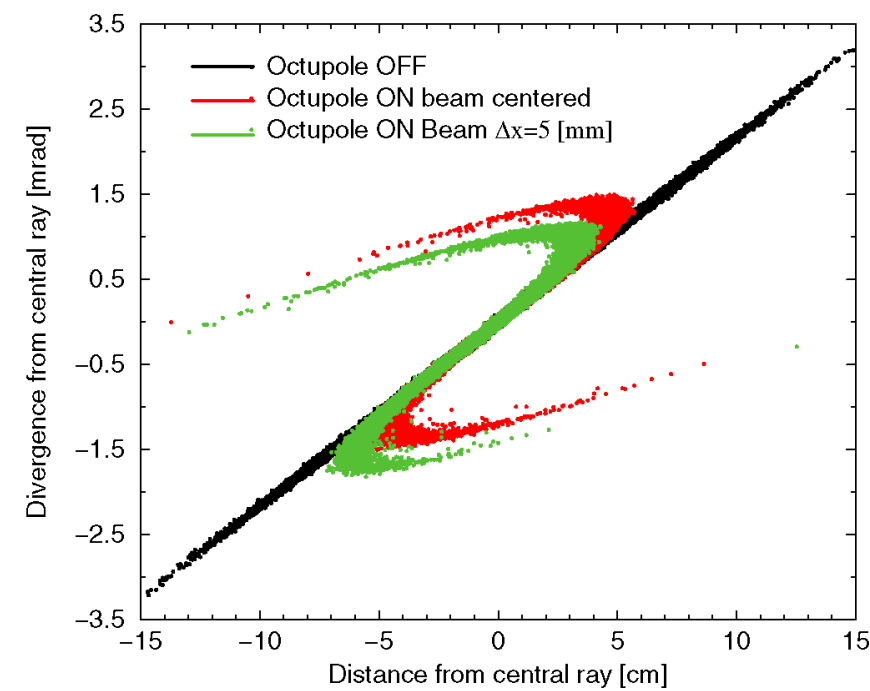

FIG. 10. (Color) Horizontal phase-space $\left(x, x^{\prime}\right)$ distributions of the beam at the target, corresponding to the settings of the horizontal octupole: (a) OFF, (b) ON, and (c) ON but misaligned with respect to the beam axis.

displaced by $5 \mathrm{~mm}$ from the beam's axis, the octupole's effect on the beam is asymmetric (green dots), causing the asymmetry of phase-space distribution $\left(x, x^{\prime}\right)$ at the target; hence, the projection of this phase-space distribution $\left(x, x^{\prime}\right)$ on the horizontal axis entails the formation of a beam with a slanted profile (green curve in Fig. 9). The calculated profile of this slanted beam is in good qualitative agreement with the measured one shown in Fig. 8 (horizontal). To explain the increase in the beam's intensity at the edges of the rectangular distributions when the octupoles are ON [Figs. 7(a)-7(d)], we note that with the octupoles OFF the phase-space distribution $\left(x, x^{\prime}\right)$ at the target is elliptical, like the distribution of the black dots in Fig. 10. Projecting such a distribution on either the $x$ or $y$ axis yields a Gaussian distribution. However, when one of the octupoles is turned ON (here only the horizontal) the phase-space distribution is "folded over" (the $x, x^{\prime}$ distributions of red dots in Fig. 10) and deviates from that of the normally distributed beam (black dots). The projection of this "folded" phase-space distribution $\left(x, x^{\prime}\right)$ on the $x$-axis generates the rectangular red curve, shown in Fig. 9, in which there is some increase in the beam's intensity at the edges of the projected distribution. This "folding" of the phase-space distribution is responsible for the increase in the beam's intensity at the edges of the projected distribution (red curve in Fig. 9), or equivalently, that at the perimeter of the beam distributions in Figs. 7(a)-7(d).

\section{BEAM COUPLING GENERATED BY OCTUPOLE ELEMENTS}

In general, exciting the octupoles causes coupling between the horizontal- and the vertical-beam coordinates, as shown by Eq. (1). However, simulations and experimental 

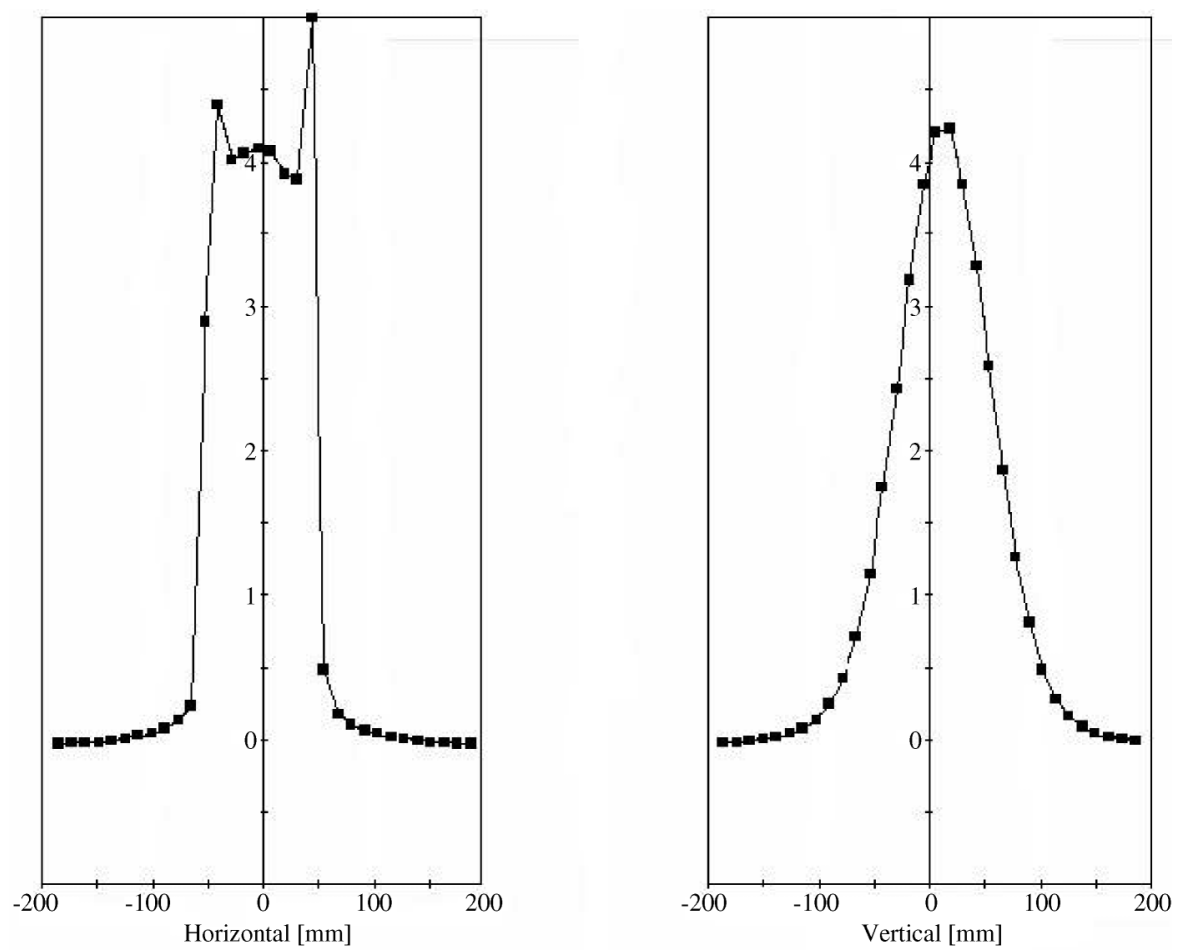

FIG. 11. Horizontal and vertical beam profiles at the target of the NSRL line with only the horizontal octupole (O1) ON. Note that only the horizontal profile is affected by the octupole $(\mathrm{O} 1)$, with the vertical profile remaining unaffected and maintaining a Gaussian shape.
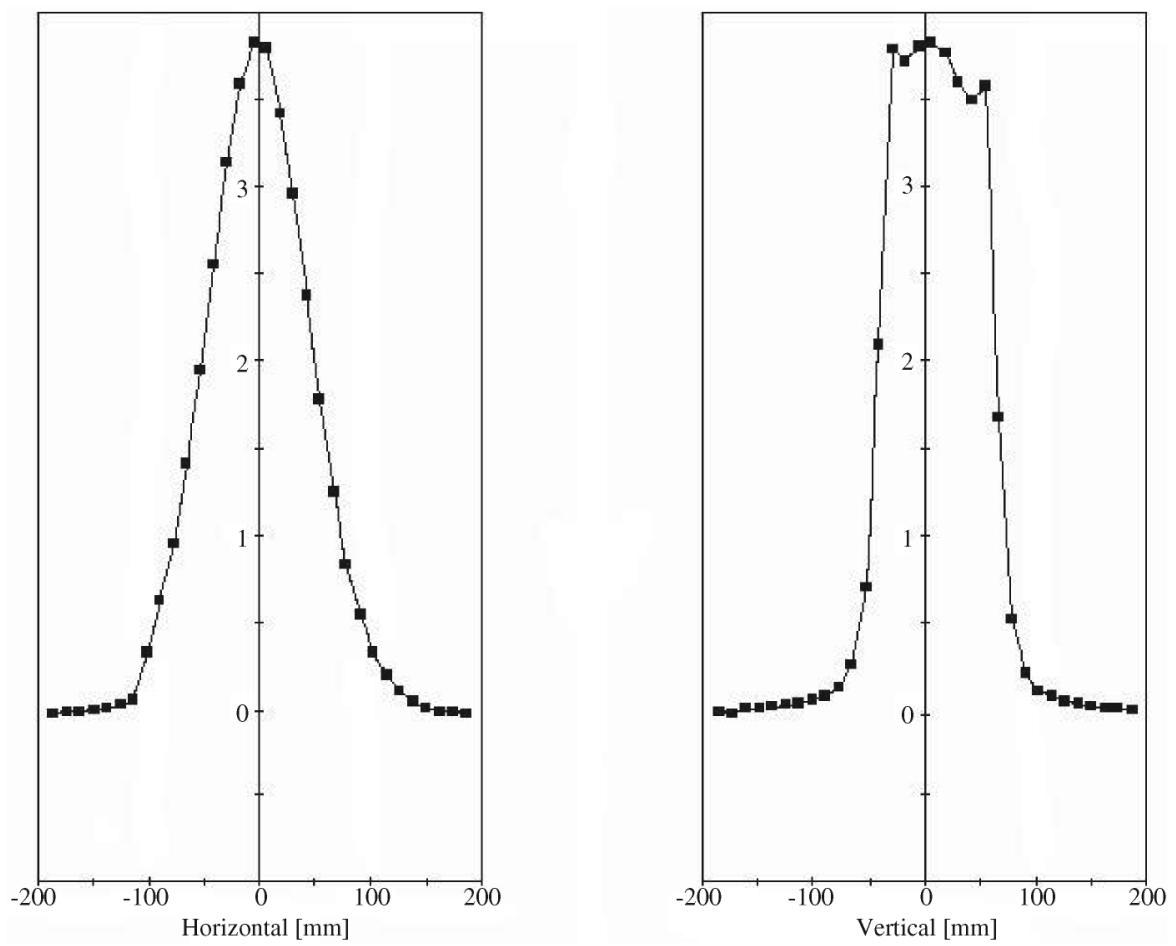

FIG. 12. Horizontal and vertical beam profiles at the target location of the NSRL line with only the vertical octupole (O2) ON. Note that only the vertical profile is affected by the octupole (O2), with the horizontal profile remaining unaffected keeping a Gaussian shape. 
measurements show that if the setup of first-order beam optics satisfies the beam constraints mentioned earlier (i.e., those that facilitate the generation of a uniform beam), then this effect is insignificant. Then, the main effect of the octupoles is a uniformly distributed rectangular beam at the target. The insignificance of the beam coupling can be demonstrated experimentally by measuring the beam profiles at the target with only one of the octupoles ON. Figure 11 shows the horizontal and vertical profiles at the target with the $\mathrm{O} 1$ octupole, nominally affecting the former, turned $\mathrm{ON}$, and the $\mathrm{O} 2$ octupole, nominally affecting the latter, turned OFF.

The nearly Gaussian shape of the vertical-beam profile in Fig. 11 denoted that the $\mathrm{O} 1$ octupole does not affect appreciably the vertical-beam profile which maintains a nearly Gaussian shape. Similarly, Fig. 12 shows the horizontal- and vertical-beam profiles at the target with the $\mathrm{O} 1$ octupole OFF and the O2 octupole ON. The nearly Gaussian shape of the former demonstrates that octupole $\mathrm{O} 2$ has little effect on it.

\section{FURTHER STUDIES}

Computer simulations, based on Eq. (3) below, show that by replacing the octupole magnetic elements with dodecapoles magnetic elements, a normal beam distribution at the target location can be transformed into a rectangular, uniform one:

$$
\begin{aligned}
X_{i}= & R_{i j} x_{j}+T_{i j k} x_{j} x_{k}+W_{i j k l} x_{j} x_{k} x_{l}+O_{i j k l m} x_{j} x_{k} x_{l} x_{m} \\
& +F_{i j k l m n} x_{j} x_{k} x_{l} x_{m} x_{n} .
\end{aligned}
$$

This equation, which is similar to Eq. (1), contains the fourth $\left(O_{i j k l m}\right)$ - and fifth $\left(F_{i j k l m n}\right)$-order aberration coefficients, because a dodecapole magnetic element is predominantly a fifth-order element. Summation is assumed over the repeated indices in each term of Eq. (3). These aberration coefficients were calculated using a modified version [7] of the RAYTRACE [8] computer code. The simulations, which are based on a "simple model" that generates the magnetic fields of a dodecapole, reveal two beneficial effects from the uniform beam distributions generated by the dodecapole magnetic elements; first, they minimize the increase in beam intensity at the perimeter of the rectangle thus affording a slightly larger area with uniform beam distribution and slightly higher beam intensity; and, second, the effect of the aberrations, causing the curved boundaries of the beam distribution at the target [Figs. 7(a)-7(c)], is less pronounced. Our future studies of the effect of the octupole and dodecapole magnetic elements on the beam's uniformity will include a more detailed investigation of the effect of the aberrations from either of these elements on the beam's distribution at the target. For these studies we will use a more realistic model of the magnetic elements that will be generated by a threedimensional code [9] for electromagnetics to provide the magnetic field of a particular magnetic element, and subsequently, a more accurate assessment of the aberration coefficients in Eqs. (1) and (3). Applying Monte Carlo calculations to these equations will give us better information about the effect of these elements on the beam's distribution at the target when they act on Gaussian or nearly Gaussian beams.

\section{SUMMARY}

In this paper we discussed our findings on the uniformity of beam distributions generated at the target location of the NSRL facility at BNL. The measured error in the beam's uniformity within the area enclosing the sample under irradiation was $\pm 2.5 \%$. We also suggested explanations of certain effects that occasionally are associated with the uniform beam distribution at the target. The quality of the beam distributions at the target proves that this method of producing uniform beam distributions compares favorably with alternative methods [10].

\section{ACKNOWLEDGMENTS}

This work was performed under the auspices of the U.S. National Aeronautics and Space Administration and the U.S. Department of Energy.

[1] N. Tsoupas, M. S. Zucker, T. E. Ward, and C. L. Snead, Jr., Nucl. Sci. Eng. 126, 71 (1997).

[2] N. Tsoupas, R. Lankshear, C. L. Snead, Jr., T. E. Ward, and M.S. Zucker, Particle Accelerator Conference, San Francisco, CA, 1991, p. 1695.

[3] N. Tsoupas, S. Bellavia, R. Bonati, K. A. Brown, I-Hung Chiang, C. J. Gardner, D. Gassner, S. Jao, A. McNerney, D. Phillips, R. Prigl, A. Rusek, and L. Snydstrup, European Particle Accelerator Conference, Lucerne, Switzerland, 2004, p. 2879.

[4] K.A. Brown, L. Ahrens, R.H. Beutenmuller, I-Hung Chiang, D. C. Elliot, D. Gassner, Z. Li, I. Marneris, J. A. Morris, D. Phillips, V. Radeka, A. Rusek, N. Tsoupas, B. Yu, and K. Zeno, European Particle Accelerator Conference, Lucerne, Switzerland, 2004, p. 1547.

[5] D. Gassner, S. Bellavia, K. A. Brown, I-Hung Chiang, P. Pile, and R. Prigl, AIP 0-7354-0103-9/02, edited by G. A. Smith and T. Russo, 2002, p. 353.

[6] D. C. Carey, SLAC-R-95-462, Fermilab-Pub-95/069 UC414.

[7] N. Tsoupas, H.A. Enge, and E. Forest, LINAC, Williamsburg, VA, 1988, p. 442.

[8] S. B. Kowalski, Nucl. Instrum. Methods Phys. Res., Sect. A 258, 407 (1987).

[9] OPERA, A computer code for Electromagnetics. Vector Fields Inc.

[10] J. Alonso, European Particle Accelerator Conference, Vienna, Austria, 2000, p. 235. 